\title{
Toll-like receptor 2 and dectin-1 function as promising biomarker for Aspergillus fumigatus infection
}

\author{
PENG-PENG ZHANG，XIAO-FENG XIN，XIAO-YONG XU，LI-PING FANG，JIE WU and YI SHI \\ Department of Respiratory and Critical Care Medicine, Jinling Hospital, Nanjing Clinical College, \\ The Second Military Medical University, Nanjing, Jiangsu 210002, P.R. China
}

Received October 10, 2016; Accepted June 2, 2017

DOI: $10.3892 /$ etm.2017.5000

\begin{abstract}
In recent years, along with the wide application of organ transplantation and immunosuppressive agents, as well as the abuse of broad spectrum antibiotics, the incidence of invasive fungal infections has been increasing gradually. The present study aimed to identify novel biomarkers in cells infected with Aspergillus fumigatus. Human umbilical vein endothelial cells (HUVECs) were infected with Aspergillus fumigatus and then harvested at different time-points $(0,1,2,4$ and $6 \mathrm{~h})$. The expression Toll-like receptor 2 (TLR2) and dectin-1 expression were examined using flow cytometry and western blotting, and fluorescence-based microscopy was used to evaluate their distribution. The results indicated that TLR2 and dectin-1 protein levels were localized on the surface of HUVECs, and that dectin-1 was distributed on HUVEC membranes as observed under confocal microscope. Immunofluorescence assay result revealed that the optical intensity of dectin-1 in the Aspergillus fumigatus-infected group was significantly increased at 0,1 and $2 \mathrm{~h}$ compared with the control group $(\mathrm{P}<0.05)$. However, the optical intensity of TLR2 in the Aspergillus fumigatus-infected group was markedly decreased between 0 and $6 \mathrm{~h}$, as compared with the control group $(\mathrm{P}<0.05)$. Western blot analysis indicated that dectin-1 expression was significantly increased and TLR2 expression was significantly decreased at 0,1 and $2 \mathrm{~h}$ post infection in the Aspergillus fumigatus-infected group compared with the control group. Furthermore, the expression of TLR2 was also negatively correlated with the concentration of Aspergillus fumigatus. In conclusion, upon infection of cells with Aspergillus fumigatus, TLR2 and dectin-1 expression levels were significantly altered. Therefore, TLR2 and dectin-1
\end{abstract}

Correspondence to: Dr Yi Shi, Department of Respiratory and Critical Care Medicine, Jinling Hospital, Nanjing Clinical College, The Second Military Medical University, 305 East Zhongshan Road, Nanjing, Jiangsu 210002, P.R. China

E-mail: ssshiyee@yeah.net

Key words: human umbilical vein endothelial cells, Aspergillus fumigatus, Toll-like receptor 2, dectin-1 levels may function as promising biomarkers for the treatment or diagnosis of Aspergillus fumigatus infection.

\section{Introduction}

In recent years, along with the wide application of organ transplantation and immunosuppressive agents, as well as the abuse of broad spectrum antibiotics, the incidence of invasive fungal infections has been gradually increasing, particularly of invasive aspergillosis (1,2). Aspergillus fumigatus is the most common pathogen of the Aspergillus genus; however, its pathogenic mechanism currently remains unclear.

The vascular endothelial cell is one of the targets of invasive infection induced by Aspergillus fumigatus. Previous in vivo and in vitro studies have demonstrated that endothelial cells can adhere or endocytose the Aspergillus fumigatus spores, resulting in increased expression of the inflammatory factors interleukin (IL)-1, tumor necrosis factor (TNF)- $\alpha$, IL-6 and IL-8 (3-5). In vascular endothelial cells, the receptor that recognizes the Aspergillus fumigatus fungus is not clear to date. As a type of pathogenic fungi, Aspergillus fumigatus can be recognized by the Toll-like receptors (TLRs) and dectin-1 molecules of the hosts in order to induce the immune reaction. To date, it has been proven that TLR2, TLR4 and dectin-1 are the main Aspergillus fumigatus-associated recognition receptors $(6,7)$

The experiments of the present study attempted to investigate the alterations in host cell-associated receptors following infection with Aspergillus fumigatus using western blot analysis and flow cytometry. The study aimed to reveal the molecules associated with the recognition of endothelial cells and Aspergillus fumigatus, as well as to provide a more comprehensive understanding of the pathogenic mechanism involved in this infection.

\section{Materials and methods}

Cell culture. Human umbilical vein endothelial cells (HUVECs; cat. no. 3425) were purchased from Nanjing KeyGen Biotech. Co.,Ltd.(Nanjing, China). Cells were cultured in the Dulbecco's modified Eagle's medium (DMEM; Invitrogen; Thermo Fisher Scientific, Inc., Waltham, MA, USA) containing $10 \%$ fetal bovine serum (Hyclone; GE Healthcare Life Sciences, Logan, UT, USA) supplement with $100 \mathrm{U} / \mathrm{ml}$ penicillin and $100 \mu \mathrm{g} / \mathrm{ml}$ 
streptomycin in a $5 \% \mathrm{CO}_{2}$ incubator under $37^{\circ} \mathrm{C}$. The present study was approved by the Ethics Committee of the Second Military Medical University (Nanjing, China).

Preparation of Aspergillus fumigatus spores and mycelium. The Aspergillus fumigatus strains were isolated and identified by Microorganism Laboratory of the Second Military Medical University. After 5 days of growth in the Sabouraud agar from Nanjing KeyGen Biotech Co., Ltd., the fungus was washed with DMEM culture solution, followed by filtering with a 16-layer gauze and centrifuging at 10,000 rpm for $3 \mathrm{~min}$ at $4^{\circ} \mathrm{C}$. The fungus with $10^{7}$ spores $/ \mathrm{ml}$ was obtained in the culture solution. Next, the spore suspension was incubated at $37^{\circ} \mathrm{C}$ and $5 \% \mathrm{CO}_{2}$ for $7 \mathrm{~h}$. Finally, the Aspergillus fumigatus spores were preserved under $4^{\circ} \mathrm{C}$.

Flow cytometry assay. The HUVECs were stimulated with $1 \mathrm{ml}\left(10^{7} / \mathrm{ml}\right)$ Aspergillus fumigatus suspension, and washed 3 times by phosphate-buffered saline (PBS) after incubation at $37^{\circ} \mathrm{C}$ for $0,1,2,4$ or $6 \mathrm{~h}$. Subsequently, the HUVECs were digested by $2 \mathrm{mM}$ EDTA and centrifuged under $750 \mathrm{x} \mathrm{g}$ for $5 \mathrm{~min}$ at room temperature. Following centrifugation, the HUVECs were then re-suspended, fixed in 3\% paraformaldehyde for $30 \mathrm{~min}$ and washed three times using PBS with centrifugation under $750 \mathrm{x}$ g for $5 \mathrm{~min}$ at room temperature. Next, the HUVECs were incubated with goat anti-human dectin-1 (1:1,000, sc-73897; Santa Cruz Biotechnology, Inc., Dallas, TX, USA) and phycoerythrin (PE)-labeled TLR2 (1:500, cat. no. 12-4752; eBioscience; Thermo Fisher Scientific, Inc.) antibodies at room temperature for $1 \mathrm{~h}$. After washing with PBS two times, the HUVECs were incubated at room temperature for $1 \mathrm{~h}$ with PE-labeled or fluorescein isothiocyanate (FITC)-labeled goat anti-mouse IgG antibodies (Santa Cruz Biotechnology, Inc.), respectively. Cells were then washed by PBS 2 times and resuspended to adjust the cell concentration to $10^{6} / \mathrm{ml}$. Finally, flow cytometry was performed and CellQuest software v3.3 (BD Biosciences, Franklin Lakes, NJ, USA) was used to observe the TLR2 and dectin-1 expression in the cells.

Western blot analysis. A total of $1 \mathrm{ml}$ Aspergillus fumigatus suspension was added to the 6-well plate seeded with HUVECs, followed by washing in cold PBS 3 times after 1, 2, 4 and 6 h of incubation. The HUVECs were collected. Next, cells were solubilized in lysis buffer containing $20 \mathrm{mM}$ Tris (pH 7.5), 135 mM NaCl, 2 mM EDTA, 2 mM DTT, 25 mM glycerophosphate, $2 \mathrm{mM}$ sodium pyrophosphate, $10 \%$ glycerol, $1 \%$ Triton $\mathrm{X}-100,1 \mathrm{mM}$ sodium orthovanadate, and $1 \mathrm{mM}$ phenylmethylsulfonyl fluoride for $1.5 \mathrm{~h}$. The lysed HUVECs were centrifuged at a speed of $8,000 \mathrm{x}$ g at $4^{\circ} \mathrm{C}$, then the pellets were removed. After quantification with $\mathrm{BCA}$, proteins in the supernatant $(20 \mu \mathrm{g} /$ lane) were separated by $12 \%$ SDS-PAGE under reducing conditions and then transferred to a polyvinylidene difluoride membrane. The membrane was blocked with 5\% skim milk for $1 \mathrm{~h}$ in TBS-Tween-20 (TBST). The membrane was washed three times with TBST (5 min/wash). The anti-TLR2 (1:1,000, cat. no. 3478; eBioscience; Thermo Fisher Scientific, Inc.) or anti-dectin-1 antibodies (1:2,000, cat. no. 12-5434; eBioscience; Thermo Fisher Scientific, Inc.) as primary antibody was added in $5 \%$ bovine serum albumin (BSA) and incubated overnight at $4^{\circ} \mathrm{C}$ on a shaker. The membrane was washed 3 times with TBST ( $5 \mathrm{~min} /$ wash). Rabbit IgG secondary antibody (1:10,000, cat. no. 3483; Thermo Fisher Scientific, Inc.) was added in TBST with 5\% skim milk for $1 \mathrm{~h}$ at room temperature. The blots were developed using a TMB immunoblotting system (cat. no. 16101200; Bio-Rad Laboratories, Inc., Hercules, CA, USA). The total density of the protein bands was calculated by using BandScan software version 4.0 (Glyko; BioMarin Pharmaceutical, Inc., San Rafael, CA, USA).

Immunofluorescence assay. A cover slip was placed in a 24-well plate (with a diameter of $1.5 \mathrm{~cm}$ ), and the HUVEC suspension with a density of 8,000 cells/well was added to the cover slip and cultured in an incubator at $37^{\circ} \mathrm{C}$ and $5 \% \mathrm{CO}_{2}$ for $24 \mathrm{~h}$. Next, $1 \mathrm{ml}\left(10^{7}\right.$ spores $\left./ \mathrm{ml}\right)$ suspension of Aspergillus fumigatus was added to the cover slip containing the cells, incubated for $\sim 4 \mathrm{~h}$ and then washed using PBS for 3 times. The cells were subsequently fixed with $3 \%$ paraformaldehyde for $30 \mathrm{~min}$, and treated with Triton X-100 for 20 min. TLR 2 and dectin- 1 antibodies were added to the cells for incubation at $4^{\circ} \mathrm{C}$ overnight, followed by incubation with the mouse or goat $\mathrm{IgG}$ fluorescence secondary antibody (1:5,000, cat. no. 3432; Thermo Fisher Scientific, Inc.) at $37^{\circ} \mathrm{C}$ for $2 \mathrm{~h}$. Finally, the cover slip was placed on a glass slide and observed under a confocal microscope.

Statistical analysis. The software SPSS version 13.0 (SPSS, Inc., Chicago, IL, USA) was used to conduct statistical analysis. The measuring data are expressed as the mean \pm standard deviation. Mean values among multiple groups were compared by the single-factor analysis of variance. $\mathrm{P}<0.05$ was considered to indicate a statistically significant difference.

\section{Results}

TLR2 and dectin-1 are distributed on the surface of the HUVECs. The distribution of the TLR2 and dectin-1 was observed using a light microscope at $4 \mathrm{~h}$ post co-incubation of Aspergillus fumigatus and HUVECs. Numerous Aspergillus fumigatus spores and longer hypha were observed in the HUVECs. In addition, green fluorescence from FITC-dectin-1 was observed around the spores and hyphae, and dectin-1 even appeared outside the cells, which indicated that the endothelial cells could be combined with the Aspergillus fumigatus. By contrast, PE-labeled TLR2 (red) only appeared inside the cells and on the cell membrane, and there was no associated red label around the Aspergillus fumigatus, which indicates that TLR2 was unable to combine with Aspergillus fumigatus. However, following stimulation by the Aspergillus fumigatus, TLR2 can be endocytosed inside the HUVECs (Fig. 1A and B).

Aspergillus fumigatus triggers the alteration of TLR2 and dectin-1 levels. In order to evaluate the expression of the TLR2 and dectin-1 proteins, the fluorescence intensity of the dye-stained proteins in the HUVECs was examined. The results indicated that the TLR2 fluorescence intensity in the Aspergillus fumigatus group was significantly decreased following incubation for 2,4 or $6 \mathrm{~h}$, compared with the control group (Fig. 2A; $\mathrm{P}<0.05$ ). The TLR2 fluorescence 
A

TLR2

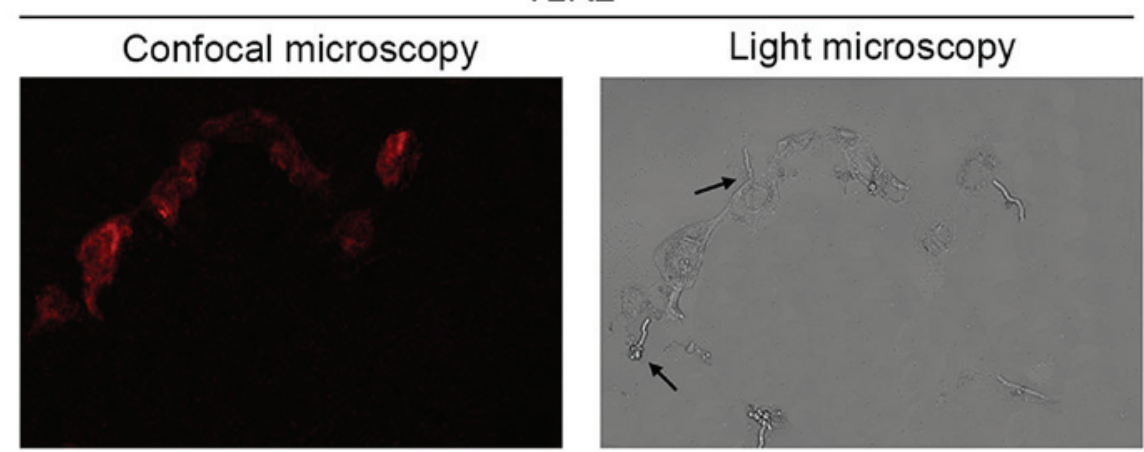

B

Dectin-1

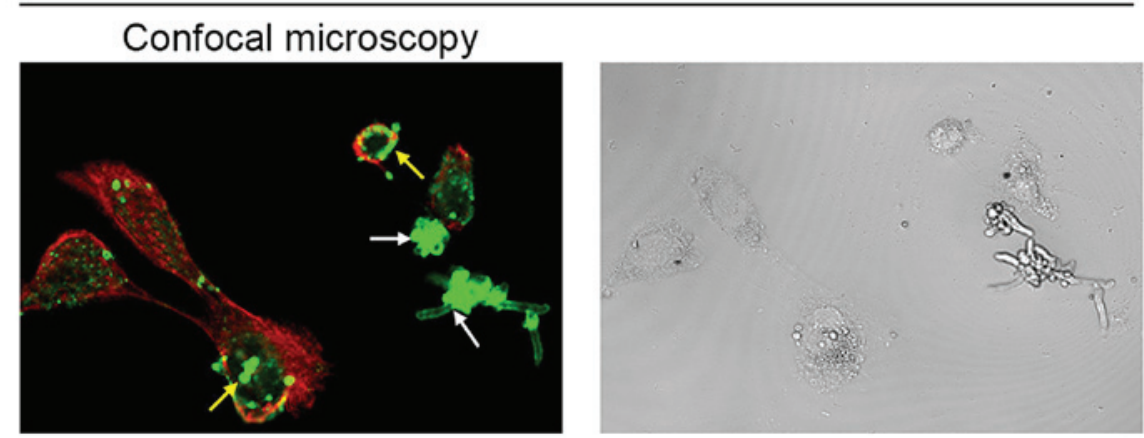

Figure 1. Expression and distribution of (A) TLR2 and (B) dectin-1 in HUVECs, observed by confocal and light microscopy. The HUVECs were incubated with Aspergillus fumigatus for $4 \mathrm{~h}$. Phycoerythrin-labeled TLR2 was observed on the surface and inside the HUVECs under confocal microscopy, while Aspergillus fumigatus hyphae (black arrow) adhered on the HUVECs under a light microscope. Dectin-1 was observed by FITC labeling (green) under confocal microscopy. The Aspergillus fumigatus conidia (yellow arrow) entered the HUVECs and green fluorescence was seen. Green fluorescence was also observed on hyphae (white arrow) outside the cells. The cytoskeleton was labeled with rhodamine-phalloidine. The same field of view was examined by both microscopy methods in each part. TLR2, Toll-like receptor 2; HUVECs, human umbilical vein endothelial cells.

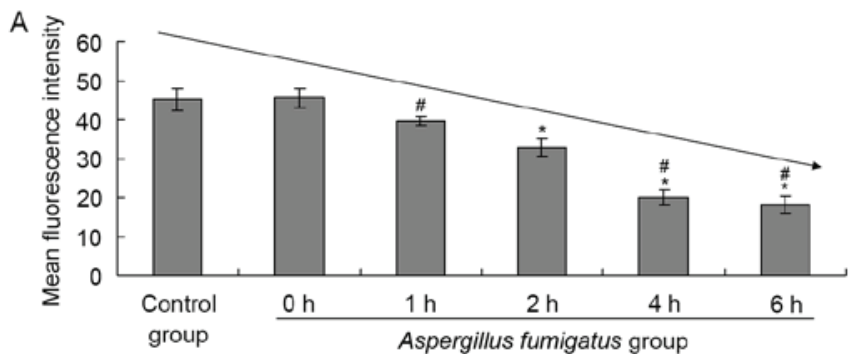

$\mathrm{B}$

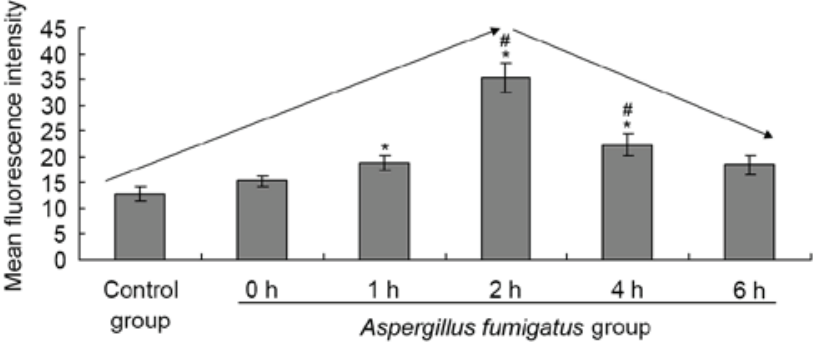

Figure 2. Mean fluorescence intensity of (A) TLR2 and (B) dectin-1. ${ }^{*} \mathrm{P}<0.05$ vs. control group; ${ }^{\#} \mathrm{P}<0.05$ vs. $2 \mathrm{~h}$ post-infection. TLR2, Toll-like receptor 2 .

intensity exhibited a time-dependent decrease, with significant differences observed between the groups (between 0 and 6 h) (Fig. 2A; all $\mathrm{P}<0.05$ ). By contrast, the dectin-1 fluorescence intensity in Aspergillus fumigatus group significantly increased at 1, 2 and $4 \mathrm{~h}$ post-infection compared with the control group (Fig. 2B; $\mathrm{P}<0.05$ ), and a peak intensity value appeared at $2 \mathrm{~h}$.

Aspergillus fumigatus infection decreases TLR2 expression and increases dectin-1 expression. The results indicated that the TLR2 expression was significantly decreased in the Aspergillus fumigatus groups as compared with the control group after 2, 4 and $6 \mathrm{~h}$ of incubation, in a time-dependent manner (Fig. 3; $\mathrm{P}<0.05$ ). As shown in the western blots, the expression of dectin-1 was most distinct following co-incubation of Aspergillus fumigatus and HUVECs for $2 \mathrm{~h}$ (Fig. 2A). The dectin-1 expression was significantly increased in the Aspergillus fumigatus groups at 1,2, 4 and $6 \mathrm{~h}$ post-infection compared with the control (Fig. 3B; $\mathrm{P}<0.05$ ). However, the dectin-1 expression was significantly reduced at 4 and $6 \mathrm{~h}$ post-infection compared with the $2 \mathrm{~h}$ post-infection with Aspergillus fumigatus (Fig. 3B; $\mathrm{P}<0.05$ ).

TLR2 expression is positively correlated with the Aspergillus fumigatus concentration. In order to investigate the promising role of the TLR2 in Aspergillus fumigatus-infected HUVECs, correlation analysis was performed in the present study. The results indicated that the TLR2 levels were positively correlated with the Aspergillus fumigatus concentrations (Fig. 4A; $\mathrm{P}<0.05$, r=0.8673). Furthermore, there was no correlation between the dectin-1 levels and the Aspergillus fumigatus concentrations (Fig. 4B; P>0.05). 


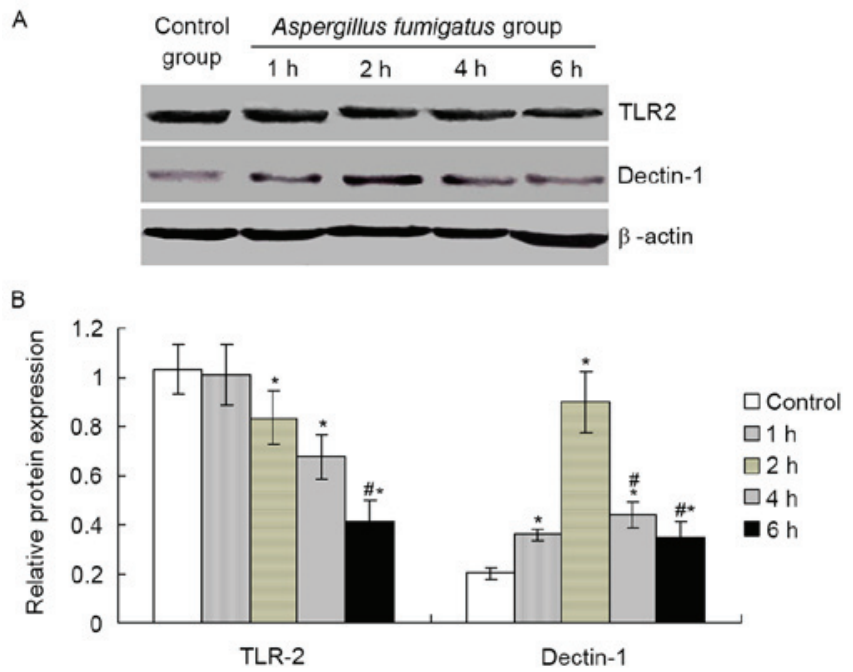

Figure 3. Expression levels of the TLR2 and dectin-1 in human umbilical vein endothelial cells incubated with Aspergillus fumigatus are demonstrated in the (A) western blots and (B) quantified results. ${ }^{*} \mathrm{P}<0.05$ vs. the control group; ${ }^{\text {P }}<0.05$ vs. $2 \mathrm{~h}$ post-infection. TLR2, Toll-like receptor 2.

\section{Discussion}

Aspergillus fumigatus has received attention due to its high mortality rate. In particular, among patients with low immunity, the mortality of invasive pulmonary aspergillosis is up to $60 \%(1,2)$. Vascular invasion is an important feature of invasive aspergillosis, and thus the interaction between the Aspergillus fumigatus and endothelial cells appears to be important. The main constituent of fungal cell wall is $\beta$-glucan, while dectin- 1 is the specific receptor of $\beta-1,3$-glucan (8). A previous in vivo experimental study demonstrated that mice with no dectin-1 expression that were nasally administered with Aspergillus fumigatus presented a significantly higher fungal load in the lung tissue as compared with wild-type mice (7). This indicates that dectin-1 serves a leading part in the process of removing Aspergillus fumigatus from the body. At the cellular level, dectin-1 is mainly expressed in the dendritic cells and mononuclear phagocytes, with reduced levels in the lymphocytes and acidophils, and no expression in the non-phagotrophic host cells, such as epithelial and endothelial cells (9). For instance, Aspergillus fumigatus stimulates the generation of IL-8 in epithelial cells independently from the TLR/MYD88/NF- $\kappa$ B pathway, but associated with the mitogen-activated protein kinase signaling pathway (10). In 2009, a Korean study observed that mycobacteria stimulated the upregulation of dectin-1 mRNA in the alveolar epithelial cells (11). In 2002, Lowe et al (12) observed that there were at least two types of glucan receptor recognition in endothelial cells. Therefore, the receptor that recognizes Aspergillus fumigatus in vascular endothelial cells remains unclear. To the best of our knowledge, no previous studies reporting the expression of dectin-1 in endothelial cells have been published to date.

The present study observed that flow cytometry was able to detect the expression of dectin-1 in endothelial cells by indirect immunofluorescence. Subsequent to culturing with Aspergillus fumigatus, dectin-1 was able to bind to the surface of Aspergillus fumigatus and be dispersed on the interior and exterior of cells. However, it was observed that the
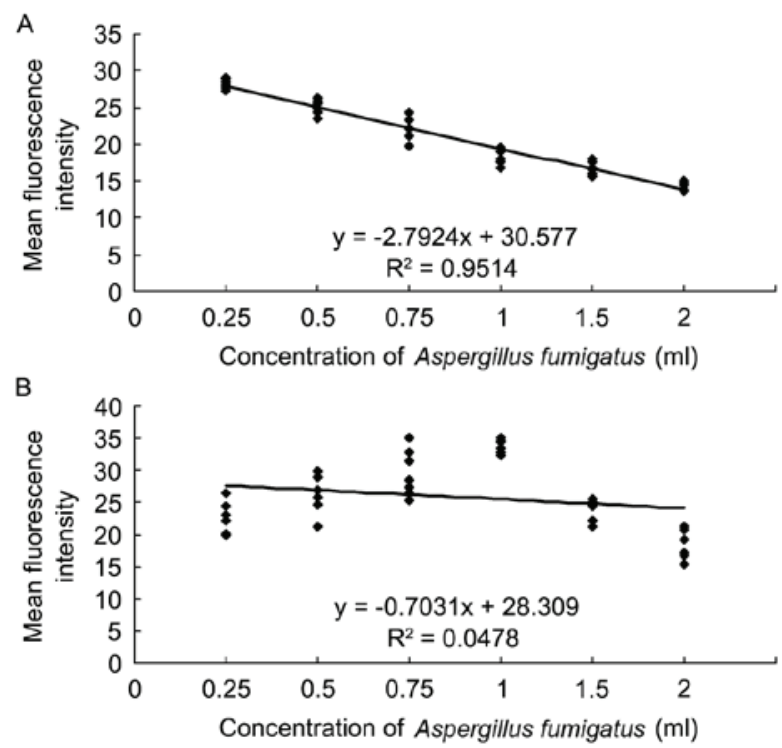

Figure 4. Correlation analysis between the concentrations of Aspergillus fumigatus and the expression levels of (A) TLR2 or (B) dectin-1. TLR2, Toll-like receptor 2 .

non-endocytosed Aspergillus fumigatus (located between the cell membrane and cell) was also able to incorporate dectin-1, which indicated that dectin-1 was secreted outside the cells. The effect of this phenomenon on the body is unknown yet, although previous findings demonstrated that dectin-1-Fc IgG was able to regulate the endocytosis process $(13,14)$.

A previous study demonstrated that TLRs serve an important role in the innate immunity (15). A mouse without TLR2 is harder to form the disseminated candidiasis compared with the wild-type one (16). TLR2 is an important receptor able to recognize the hypha and spores of Aspergillus fumigatus. With the aid of flow cytometry, the present study detected the expression of membrane receptor TLR2 at different time-points following the stimulation of the endothelial cells with Aspergillus fumigatus. Following prolonged contact with Aspergillus fumigatus, TLR2 expression exhibited a gradually descending trend. However, previous research indicated that TLR2 could enter into macrophages (17). According to confocal microscopy examination in the present study, TLR2 was observed in the endothelial cells, however, it did not appear to combine with Aspergillus fumigatus. Furthermore, the Aspergillus fumigatus surface at the exterior of the cell displayed no fluorescence for TLR2. The reason underlying this effect may be that, although TLR2 is able to recognize Aspergillus fumigatus, the recognition does not mean also combination. A previous study suggested that in macrophages without TLR2 and/or TLR4 expression, the capacity for binding with the spores of Aspergillus fumigatus was not altered (18).

The recognition ligand of TLRs remains unclear, although Aspergillus fumigatus secretes a great variety of proteins, including certain important pathotoxins, such as gliotoxin. TLR2 may recognize or bind to certain secretory proteins in order to enter into the cells. Therefore, further study is required on the specific ligand of Aspergillus fumigatus for its recognition by TLRs. Additionally, a certain time is necessary from the transcription to the translation, and to the expression of proteins, and studies at the protein level are unable to report 
the entire process of receptor alterations. A previous study identified that the spores of Aspergillus fumigatus stimulated the mononuclear cell membrane in the peripheral blood, while the expression of receptor TLR2 was decreased, but the mRNA level of TLR2 was not affected (19). Accordingly, further study on the influence of Aspergillus fumigatus on the endothelial cell receptor TLR2 should be performed.

TLR 2 and dectin-1 are the well-characterized biomarkers for the recognition of Aspergillus fumigatus, according to previous studies (20-24). These studies mainly explored the TLR2 and dectin-1 levels in patients and animals. However, the present study aimed to identify a novel biomarker for quick examination of this pathogen in clinical practice. Therefore, the application of the TLR2 and dectin-1 in HUVECs was mainly explored by detecting the fluorescence intensity. Meanwhile, the present study also investigated the correlation between TLR2 or dectin-1 and the Aspergillus fumigatus concentrations. Therefore, novel results were reported herein, in comparison with previous studies (20-24).

In conclusion, the results of the present study experiments indicated that, following incubation with Aspergillus fumigatus, the expression and distribution of dectin-1 and TLR2 in HUVECs were significantly altered, and the expression of TLR2 was also negatively correlated with the concentration of Aspergillus fumigatus. Thus, TLR2 and dectin-1 levels may function as promising biomarkers for the diagnosis of Aspergillus fumigatus infection.

\section{Acknowledgements}

The authors are grateful to Dr. Li-Jun Zhang (Nanjing University, Nanjing, China) for assistance with confocal microscopy, and all the staff members of the Department of Respiratory Medicine (Nanjing General Hospital of Nanjing Military Command, Nanjing, China).

\section{References}

1. Li RF, Lu YL, Lu YB, Zhang HR, Huang L, Yin Y, Zhang L, Liu S, Lu Z and Sun Y: Antiproliferative effect and characterization of a novel antifungal peptide derived from human Chromogranin A. Exp Ther Med 10: 2289-2294, 2015.

2. Walsh TJ, Anaissie EJ, Denning DW, Herbrecht R, Kontoyiannis DP, Marr KA, Morrison VA, Segal BH, Steinbach WJ, Stevens DA, et al: Treatment of aspergillosis: Clinical practice guidelines of the infectious diseases society of America. Clin Infect Dis 46: 327-360, 2008.

3. Paris S, Boisvieux-Ulrich E, Crestani B, Houcine O, Taramelli D, Lombardi L and Latgé JP: Internalization of Aspergillus fumigatus conidia by epithelial and endothelial cells. Infect Immun 65 $1510-1514,1997$.

4. Ibrahim-Granet O, Philippe B, Boleti H, Boisvieux-Ulrich E, Grenet D, Stern M and Latgé JP: Phagocytosis and intracellular fate of Aspergillus fumigatus conidia in alveolar macrophages. Infect Immun 71: 891-903, 2003.

5. Chiang LY, Sheppard DC, Gravelat FN, Patterson TF and Filler SG: Aspergillus fumigatus stimulates leukocyte adhesion molecules and cytokine production by endothelial cells in vitro and during invasive pulmonary disease. Infect Immun 76: 3429-3438, 2008.

6. Bellocchio S, Montagnoli C, Bozza S, Gaziano R, Rossi G, Mambula SS, Vecchi A, Mantovani A, Levitz SM and Romani L: The contribution of the Toll-like/IL-1 receptor superfamily to innate and adaptive immunity to fungal pathogens in vivo. J Immunol 172: 3059-3069, 2004.

7. Werner JL, Metz AE, Horn D, Schoeb TR, Hewitt MM, Schwiebert LM, Faro-Trindade I, Brown GD and Steele C: Requisite role for the dectin-1 beta-glucan receptor in pulmonary defense against Aspergillus fumigatus. J Immunol 182: 4938-4946, 2009.
8. Adams EL, Rice PJ, Graves B, Ensley HE, Yu H, Brown GD, Gordon S, Monteiro MA, Papp-Szabo E, Lowman DW, et al: Differential high-affinity interaction of dectin-1 with natural or synthetic glucans is dependent upon primary structure and is influenced by polymer chain length and side-chain branching. J Pharmacol Exp Ther 325: 115-123, 2008.

9. Taylor PR, Brown GD, Reid DM, Willment JA, Martinez-Pomares L, Gordon S and Wong SY: The beta-glucan receptor, dectin-1, is predominantly expressed on the surface of cells of the monocyte/macrophage and neutrophil lineages. J Immunol 169: 3876-3882, 2002.

10. Balloy V, Sallenave JM, Wu Y, Touqui L, Latgé JP, Si-Tahar M and Chignard M: Aspergillus fumigatus-induced interleukin-8 synthesis by respiratory epithelial cells is controlled by the phosphatidylinositol 3-kinase, p38 MAPK, and ERK1/2 pathways and not by the toll-like receptor-MyD88 pathway. J Biol Chem 283: 30513-30521, 2008.

11. Lee HM, Yuk JM, Shin DM and Jo EK: Dectin-1 is inducible and plays an essential role for mycobacteria-induced innate immune responses in airway epithelial cells. J Clin Immunol 29: 795-805, 2009.

12. Lowe EP, Wei D, Rice PJ, Li C, Kalbfleisch J, Browder IW and Williams DL: Human vascular endothelial cells express pattern recognition receptors for fungal glucans which stimulates nuclear factor kappaB activation and interleukin 8 production. Winner of the Best Paper Award from the Gold Medal Forum. Am Surg 68: 508-518, 2002.

13. Mattila PE, Metz AE, Rapaka RR, Bauer LD and Steele C: Dectin-1 Fc targeting of Aspergillus fumigatus beta-glucans augments innate defense against invasive pulmonary aspergillosis. Antimicrob Agents Chemother 52: 1171-1172, 2008.

14. Rapaka RR, Goetzman ES, Zheng M, Vockley J, McKinley L, Kolls JK and Steele C: Enhanced defense against Pneumocystis carinii mediated by a novel dectin-1 receptor Fc fusion protein. J Immunol 178: 3702-3712, 2007.

15. Harzenetter MD, Novotny AR, Gais P, Molina CA, Altmayr F and Holzmann B: Negative regulation of TLR responses by the neuropeptide CGRP is mediated by the transcriptional repressor ICER. J Immunol 179: 607-615, 2007.

16. Netea MG, Sutmuller R, Hermann C, Van der Graaf CA, Van der Meer JW, van Krieken JH, Hartung T, Adema G and Kullberg BJ: Toll-like receptor 2 suppresses immunity against Candida albicans through induction of IL-10 and regulatory T cells. J Immunol 172: 3712-3718, 2004.

17. Underhill DM, Ozinsky A, Hajjar AM, Stevens A, Wilson CB, Bassetti $\mathrm{M}$ and Aderem A: The Toll-like receptor 2 is recruited to macrophage phagosomes and discriminates between pathogens. Nature 401: 811-815, 1999.

18. Luther K, Torosantucci A, Brakhage AA, Heesemann J and Ebel F: Phagocytosis of Aspergillus fumigatus conidia by murine macrophages involves recognition by the dectin-1 beta-glucan receptor and Toll-like receptor 2. Cell Microbiol 9: 368-381, 2007.

19. Chai LY, Kullberg BJ, Vonk AG, Warris A, Cambi A, Latgé JP, Joosten LA, van der Meer JW and Netea MG: Modulation of Toll-like receptor 2 (TLR2) and TLR4 responses by Aspergillus fumigatus. Infect Immun 77: 2184-2192, 2009.

20. Heenekamp T, Schmidt H, Lapp K, Pähtz V, Shopova I, Köster-Eiserfunke N, Krüger T, Kniemeyer O and Brakhage AA: Interference of Aspergillus fumigatus with the immune response. Semin Immunopathol 37: 141-152, 2015.

21. Curty N, Kubitschek-Barreira PH, Neves GW, Gomes D, Pizzatti L, Abdelhay E, Souza GH and Lopes-Bezerra LM: Discovering the infectome of human endothelial cells cehallenged with Aspergillus fumigatus applying a mass spectrometry label-free approach. J Proteomics 97: 126-140, 2014.

22. Netea MG, Warris A, Van der Meer JW, Fenton MJ, Verver-Janssen TJ, Jacobs LE, Andresen T, Verweij PE and Kullberg BJ: Aspergillus fumigatus evades immune recognition during germination through loss of toll-like receptor- 4 mediated signal transduction. J Infect Dis 188: 320-326, 2003.

23. Balloy V, Si-Tahar M, Takeuchi O, Philippe B, Nahori MA, Tanguy M, Huerre M, Akira S, Latgé JP and Chignard M: Involvement of toll-like receptor 2 in exprerimental invasive pulmonary aspergillosis. Infect Immun 73: 5420-5425, 2005.

24. Werner JL, Metz AE, Horn D, Schoeb TR, Hewitt MM, Schwiebert LM, Faro-Trindade I, Brown GD and Steele C: Requisite role for th dectin-1 beta-glucan receptor in pulmonary defense against Aspergillus fumigatus. J Immunol 182: 4938-4946, 2009. 